\title{
Aplicaciones de Software Científico para el análisis de datos en diseños mixtos de investigación.
}

\section{Applications of Scientific Software for the analysis of data in mixed research designs}

\author{
Aplicações de Software Científico para a análise de dados em projetos de pesquisa mistos
}

\author{
Edixon Javier Chacón-Guerrero ${ }^{\mathrm{a}^{*}}$, Rolando Antonio Eslava-Zapata ${ }^{\mathrm{b}}$

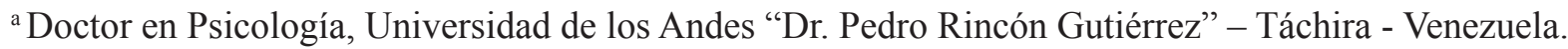 \\ Orcid: 0000-0003-1519-5456
}
${ }^{\mathrm{b}}$ Doctor en el programa de Nuevas tendencias en administración de organizaciones, Universidad de los Andes "Dr. Pedro Rincón Gutiérrez" - Táchira - Venezuela. Orcid: 0000-0003-2581-1873

Forma de citar: Chacón, E., Eslava, R. (2017). Aplicaciones de Software Científico para el análisis de datos en diseños mixtos de investigación. Eco matemático 8(1). 106-115

Recibido: noviembre 6 de 2016

Aceptado: diciembre 27 de 2016

\section{Palabras clave}

Software Científico, Datos cualitativos, Datos cuantitativos, Complementariedad, Diseños mixtos de investigación, Triangulación de datos.

\section{Keywords}

Scientific Software, Qualitative data, Quantitative data, Complementarity, Mixed research designs, Data triangulation.
Resumen: El presente artículo reflexiona sobre el uso del software científico como herramienta fundamental para el análisis de los datos en la investigación de las ciencias sociales. El artículo contiene tres partes claramente diferenciadas. La primera parte aborda la naturaleza de los datos en la investigación en las ciencias sociales. En la segunda parte se examina la importancia del software científico en el análisis de datos. En la tercera parte se presentan algunas aplicaciones con softwares científicos usados en la investigación cuantitativa, cualitativa y mixta. Las reflexiones realizadas permiten identificar que la investigación en las ciencias sociales tiene una gran oportunidad de desarrollo con el apoyo del software científico, sobre todo, para dar validez y precisión en los resultados del estudio. Existe aún todavía un largo camino por recorrer a fin de que la comunidad investigadora haga uso del software científico para el análisis de datos y lo adopte como una herramienta obligatoria a fin de fomentar el desarrollo de la investigación en ciencias sociales.

\begin{abstract}
Este artigo reflete sobre o uso do software científico como uma ferramenta fundamental para a análise de dados na pesquisa em ciências sociais. $\mathrm{O}$ artigo contém três partes claramente diferenciadas. A primeira parte aborda a natureza dos dados em pesquisas nas ciências sociais. A segunda parte examina a importância do software científico na análise de dados. A terceira parte apresenta algumas aplicações com softwares científicos utilizados em pesquisas quantitativas, qualitativas e mistas. As reflexões feitas nos permitem identificar que a pesquisa nas ciências sociais tem uma grande oportunidade de se desenvolver com o apoio do software científico, acima de tudo, para dar validade e precisão nos resultados do estudo. Há ainda um longo caminho a ser percorrido para que a comunidade de pesquisa possa fazer uso de softwares científicos para análise de dados e adotá-los como uma ferramenta obrigatória, a fim de incentivar o desenvolvimento de pesquisas em ciências sociais.
\end{abstract}

\footnotetext{
* Autor para correspondencia edixon@yahoo.com

dx.doi.org/10.22463/17948231.1481
} 


\section{Palavras-chave}

Software Científico, Dados Qualitativos, Dados Quantitativos, Complementarida de, Desenhos de pesquisa mista, Triangulação de dados.
Resumo: Este artigo reflete sobre o uso do software científico como uma ferramenta fundamental para a análise de dados na pesquisa em ciências sociais. O artigo contém três partes claramente diferenciadas. A primeira parte aborda a natureza dos dados em pesquisas nas ciências sociais. A segunda parte examina a importância do software científico na análise de dados. A terceira parte apresenta algumas aplicações com softwares científicos utilizados em pesquisas quantitativas, qualitativas e mistas. As reflexões feitas nos permitem identificar que a pesquisa nas ciências sociais tem uma grande oportunidade de se desenvolver com o apoio do software científico, acima de tudo, para dar validade e precisão nos resultados do estudo. Há ainda um longo caminho a ser percorrido para que a comunidade de pesquisa possa fazer uso de softwares científicos para análise de dados e adotá-los como uma ferramenta obrigatória, a fim de incentivar o desenvolvimento de pesquisas em ciências sociais.

\section{Introducción}

Con el pasar del tiempo las disciplinas en las ciencias sociales se han ido consolidando a la luz de las transformaciones socio-políticas-económicasculturales de los países. La producción de conocimiento en las ciencias sociales ha tomado gran importancia dada la demanda creciente por las carreras y el interés por mejorar la disciplina. De esta manera las metodologías y el desarrollo de la investigación social toma importancia, en miras de contribuir al desarrollo del conocimiento. La nueva época, caracterizada por la sociedad del conocimiento procura el fortalecimiento de la actividad investigativa siguiendo lineamientos adecuados que deriven en la producción de conocimientos. Bajo esta misma lógica, las propuestas epistemológicas, las cuales plantean la producción de conocimiento a partir del estudio de constructos, sugieren la construcción de teorías con el apoyo del software científico como un camino para generar investigación y dar cuenta del uso de un enfoque metodológico inherente a las ciencias sociales.

El análisis de datos apoyados en los softwares científicos ha llegado para quedarse (Hernández Sánchez, Borjón Robles \& Torres Ibarra, 2016), y en este momento atraviesa momentos trascendentales dado que su uso por los investigadores ha experimentado un notable incremento en todo el mundo, o bien por las características del software o bien por las funcionalidades que ofrecen, puesto que facilitan el proceso de análisis. Hoy día se da un paralelismo entre las metodologías cuantitativas, cualitativas y mixtas con la tecnología; dada la imbricación que se da entre los softwares científicos y la metodología, que ha terminado por transformar las prácticas de investigación, ofreciendo un sinfín de actividades y redefiniendo la forma de generar el conocimiento.

El uso del software científico en el análisis de datos ofrece una gran diversidad de posibilidades para las tareas analíticas (Pabón-Gómez, 2014). Hay que tener presente que antes de utilizar cualquiera de los softwares en el proceso de análisis, hay que definir claramente el diseño de la investigación para luego la elegir el software. También, hay que tomar en cuenta el tipo de materiales que pueden analizarse con el programa (números, texto, imagen, audio y vídeo) y las operaciones básicas que el paquete permite realizar (codificación y recuperación, anotaciones, construcción de modelos, entre otros). Asimismo, hay que valorar la flexibilidad de la interfase (el uso de menús contextuales o las opciones para visualizar los elementos elaborados en el análisis). También sería necesario tomar en cuenta los sistemas operativos en los que funciona el software y, en un plano más general, la complejidad de su manejo.

El presente artículo reflexiona sobre el uso del software científico como herramienta fundamental para el análisis de los datos en la investigación de las ciencias sociales. El cuerpo del artículo está 
estructurado en tres secciones. La primera de ellas introduce a la investigación en las ciencias sociales. La siguiente sección se centran en el análisis del software científico y su vinculación con el análisis de los datos de las investigaciones. La última sección introduce diversos aspectos de algunos softwares científicos usados en la investigación cuantitativa, cualitativa y mixta.

\section{Resultados y discusión}

\section{Investigación en las Ciencias Sociales}

La investigación en las ciencias sociales busca facilitar la comprensión de un caso de estudio que pasa por distintos momentos como son el epistémico, el teórico, el metodológico y el de comunicación (Bonilla Castro, Hurtado Prieto \& Jaramillo Herrera, 2009). La metodología de la investigación supone el paso por distintas fases que se apoya en técnicas a fin de abordar científicamente la realidad estudiada. El investigador debe reconocer la complejidad del estudio y precisar de herramientas científicas que le permitan analizar los datos de manera objetiva y precisa (Herrera, 2010).

El momento epistémico se inicia con la escogencia deuntemaquelepermitadesarrollarlainvestigación (Abello Llanos, 2008a). Considerando que la investigación debe incrementar el volumen de conocimiento es preciso justificarla desde el punto de vista técnico; es decir, sobre la conveniencia y relevancia de la investigación (Hernández Sampieri, Fernández Collado \& Baptista Lucio, 2006). También, debe justificarse desde el punto de vista teórico; es decir, el aporte al conocimiento (Sabino 1996). Finalmente, el aporte metodológico; es decir, si la investigación va ayudar a crear una nueva forma de obtener y analizar los datos; es aquí, donde entran a ocupar un papel importante el software científico (Abello Llanos, 2009). El aporte académico, debe considerar el estado de conocimiento actual con el propósito de generar categorías teóricas y conceptuales basadas en herramientas técnicas para dar respuesta certera al problema en estudio (Jiménez Becerra, 2004).
En este momento epistémico se ubica el problema de investigación $\mathrm{y}$, el investigador debe estar en capacidad de identificar las categorías y las variables de estudio, de ahí, que hay que examinar con cuidado lo referente a la validez y confiabilidad del estudio (Cazau, 2006). La operacionalización de las variables se hace presente, como una herramienta que va a permitir identificar los constructos que se investigan (Briones, 1998). La conceptualización teórica de la variable es un paso importante de la investigación, dado que permite ubicar el estado del arte de los enfoques teóricos y las categorías existentes. A partir de este punto, se hace necesario precisar el equilibrio teórico y metodológico de la investigación y buscar la forma como serán medidas variables. El software científico permitirá al investigador resolver de manera coherente la tarea de validación y confiabilidad de las pruebas de medición del fenómeno estudiado.

El momento teórico supone una revisión exhaustiva del conocimiento ligado al caso de estudio (Abello Llanos, 2008b) y, la búsqueda de información empírica permitirá conceptualizar las variables en estudio (Balestrini, 1998). En esta fase se establece la postura teórica y conceptual de la investigación apoyados en el manejo de las teorías, la revisión empírica segundaría y primaria a fin de fundamentar el problema de estudio (Mejía Correa, 2009). El proceso de argumentación abre paso al planteamiento de las posibles hipótesis de la investigación (Hernández Sampieri, Fernández Collado y Baptista Lucio, 2006).

En cuanto al momento metodológico, es necesario que el investigador plantee el tipo y diseño de la investigación, la población y muestra, las técnicas o instrumentos de recolección de datos y la validez y confiabilidad de las técnicas o instrumentos, con el mayor rigor científico. En esta fase, hace su presencia el software científico, dado que el investigador debe dar respuesta a las interrogantes de investigación con un diseño metodológico coherente y un procedimiento científico fundamentado indispensable para explicar el problema y el logro de los objetivos del trabajo. 
Sea cual sea el método, cualitativo, cuantitativo o mixto, hay que escoger el software científico adecuado, a fin de dar una estructura lógica al enfoque metodológico y a las subsiguientes etapas de análisis e interpretación de los resultados. Ahora bien, haciendo referencia a los resultados, conviene precisar cómo se van a procesar e interpretar los resultados y a las mediciones a las que se pretende llegar.

\section{Software Científico y Análisis de Datos}

En el campo de la investigación cualitativa se ha procurado ser más elocuentes en los procesos de análisis de datos detallando procedimientos y sus fundamentos con el fin de garantizar la sistematicidad y rigor del estudio. Sin embargo, las metodologías disponibles en la literatura científica y académica no deben tomarse como reglas infalibles y/o algoritmos estandarizados, las decisiones del investigador a nivel del diseño del estudio forman parte central de la lógica científica para dar respuesta a las preguntas e hipótesis de la investigación.

Aunque muchos investigadores tengan clara la existencia de objetos de investigación cuyos planteamiento deben abordarse estrictamente desde métodos cuantitativo o desde el punto de vista netamente cualitativo, también existe una tendencia importante hacia la consideración e implementación de diseños mixtos de investigación, los cuales, sin duda, contribuyen a fortalecer la aproximación a la realidad del objeto de estudio para poder dar respuesta a las interrogantes y objetivos de la investigación.

Distintos autores han tratado esta integración de métodos, Polit \& Hungler (1997) plantean la complementariedad de métodos como una mutua aportación entre perspectivas metodológicas. Por otra parte, autores como Rodríguez, 2003; Chacón (2003;2016) plantean diferentes interacciones entre los procedimientos analíticos de análisis de datos directamente relacionados la versatilidad y potencialidad de los diversos paquetes de software las estrategias de integración. Chacón (2016) considera tres estrategias básicas en el tratamiento de datos de naturaleza mixta en la investigación: derivación, acoplamiento y triangulación de datos.

La derivación se refiere a la obtención de datos numéricos a partir de datos textuales, por ejemplo, la obtención de tablas de frecuencias a partir del análisis bajo codificación teórica de entrevistas o grupos de discusión. El acoplamiento consiste en la consideración de un conjunto de datos, indistintamente de su naturaleza cuantitativo o cuantitativa, como un cuerpo único sobre el cual se aplican estrategias y procedimientos analíticos y se obtienen un resultado global. Por ejemplo, el análisis en redes sociales, los datos pueden tener información estructurada (numérica) con alguna breve narrativa explicativa (cualitativa). Con un enfoque más clásico se encuentre la triangulación de datos se basa en el establecimiento de relaciones de resultados de diversa naturaleza con el fin de proporcionar una base de convergencia para la identificación de elementos teóricos o el establecimiento de vínculos.

En función de las estrategias planteadas es importante reflexionar sobre los distintos escenarios ofertados por el mercado de herramientas que nos presentan cada día, considerando al mismo tiempo, las tendencias integralistas en el análisis de datos en diseños mixtos de investigación en las ciencias sociales. A continuación, se exponen algunas aplicaciones en el uso de estas estrategias basadas en los trabajos de Chacón \& Chacón (2010), Carrasquero \& Chacón (2010).

\section{Software Científico en la Investigación Cuantitativa, Cualitativa y Mixta}

\section{Lisrel}

Es un paquete de aplicaciones usado en la investigación cuantitativa que permite contrastar empíricamente modelos teóricos recursivos y no recursivos a través de las ecuaciones lineales estructurales (Cupani, 2012). Lisrel es un software creado por los profesores Karl Jöreskog y Dag Sörbom, adscritos a la Universidad de Uppsala, en 
Suecia. El software permite estimar parámetros de ecuaciones estructurales a fin de probar la validez y la adecuación del modelo estructural planteado. Lisrel (Jöreskog \& Sörbom, 1996) y Lisrel interactivo (Du Toit \& Du Toit, 2001), facilita el cálculo de los parámetros de un modelo lineal estructural. También existen otros programas que ofrecen estas y otras posibilidades tales como son: EQS (Bentler \& Wu, 1995), AMOS (Arbuckle, 1995), RAMONA o SEPATH.

La medición de las variables (análisis de factores confirmatorio) y las relaciones que se definen entre ellas (regresión) se logra por medio de la sintaxis del software (Escobedo Portillo, Hernández Gómez, Estebané Ortega \& Martínez Moreno, 2016). Asimismo, se pueden calcular los errores de medición y estimar relaciones libres de error entre las variables (Ruiz, Pardo \& San Martín, 2010). Lisrel ofrece la posibilidad de examinar una estructura en grupos diferentes $\mathrm{y}$, analizar la influencia de variables moderadoras tales como el sexo o la edad. Las versiones más recientes (i.e., LISREL 8.7) están disponibles para la plataforma Windows, por ejemplo, Prelisrel es un pre-procesador para Lisrel usado en análisis preliminares (data screening) de los datos recolectados, su distribución normal, transformaciones, generación de matrices, entre otros. Lisrel permite evaluar modelos lineales estructurales usando la sintaxis Lisrel; mientras que, Lisrel interactivo, usa el método conocido como point-and-click en una serie de ventanas. El software posee una notación propia para las variables y las matrices. Al respecto, las letras griegas en mayúscula se refieren a las variables latentes y matrices; mientras que, las letras griegas en minúscula identifican los vectores, errores de medición, entre otros.

Normalmente el software muestra las relaciones entre las variables latentes (encerradas en los círculos más grandes) y las variables observadas (encerradas en los rectángulos) con el error de medición (encerrados en los círculos más pequeños). En este sentido, las líneas grandes con una sola flecha y que unen a las variables latentes $(\beta, \gamma)$ representan la dirección de la relación causal. Por su lado, las curvas con doble flecha son las correlaciones o covarianzas $(\varphi, \psi)$. Las flechas más pequeñas que van de las variables latentes hacia las variables observadas tienen los indicadores $\lambda$, esto es el modelo de medición. La proporción de la varianza no explicada para las variables observadas está dada por el símbolo $\delta$ (delta) en el caso de las " $\mathrm{x}$ " $y$, en el caso de las "y" está dada con el símbolo $\varepsilon$ (epsilon). En cuanto a las variables latentes, la varianza no explicada viene dada con el símbolo $\zeta$ (zeta).

En el sistema de ecuaciones las variables son de dos clases, observadas directamente y las latentes (no son observadas); sin embargo, están relacionadas con las primeras. Lisrel considera que existe una estructuración casual entre las variables latentes y las variables observadas. El software consta de dos partes: el modelo estructural (establece las relaciones existentes entre las variables latentes) y el modelo de medida (establece las relaciones de las variables latentes con los indicadores).

\section{Atlas.ti}

El Atlas.ti es un software usado en el análisis cualitativo (Cárdenas Barrios, \& González Ferro, 2016), que facilita la organización, manejo e interpretación de información textual, tales como textos, imágenes, sonidos, mapas y/o videos. Algunos aspectos importantes del software se hallan en:

- Visualización: el software se adapta a la forma en la que los humanos pensamos y encontramos soluciones (sistemática, flexible y creativa). Asimismo, el Atlas.ti permite la visualización rápida y organizada de grandes cantidades de información (Blanco Álvarez, 2012).

- Integración: el Atlas.ti permite trabajar de la manera como se requiera sea de forma general o específica (Ruiz Palmero, 2011).

- Serendipity: el software facilita el 
descubrimiento de cosa, sin necesariamente haberlas estado buscándolas; esto se debe a que el Atlas.ti se adapta a la forma en la que las personas buscan la información; es decir, el software evoluciona con las personas durante el proceso investigación (Sabariego Puig, Vilà Baños \& Sandín Esteban, Julio).

- Exploración: el software provee a las personas de herramientas para indagar según sus necesidades. El análisis cualitativo se puede llevar a cabo de forma organizada y rigurosa, sin dejar a un lado la flexibilidad que requiere el proceso investigativo (San Martín, 2014).

El Atlas.ti es un software muy versátil y flexible que permite realizar el análisis cualitativo de una forma simple (Servio, 2016). El proceso comienza con la creación de una unidad hermenéutica (Varguillas, 2006), para luego asignar los documentos primarios. Seguidamente se descubren los segmentos relevantes y se crean los códigos y anotaciones. Finalmente se construye la teoría y relacionan los conceptos para elaborar el documento contentivo del resultado de la investigación. El Atlas.ti puede agruparse en un nivel textual y un nivel conceptual. El nivel textual comprende la preparación y el manejo de los datos, la segmentación de los textos y la codificación. Por su lado, el nivel conceptual comprende la comparación e interpretación de segmentos ya codificados; asimismo, la elaboración de redes a fin de llevar los conceptos (documentos primarios, citas, códigos, familias, entre otros) a un nivel mucho más abstracto y lograr la construcción de la teoría fundamentada en los datos. Cabe destacar que el investigador puede pasar del nivel textual al nivel conceptual o viceversa, todo depende de los requerimientos del mismo proceso investigativo.

En este sentido, el nivel textual requiere pasar por las siguientes etapas:

- Crear una unidad hermenéutica (archivo que contiene toda la información: datos, anotaciones, códigos, redes, entre otros.
- Asignar los documentos primarios que se van a analizar en un mismo archivo (la unidad hermenéutica).

- Leer los textos a fin de identificar y marcar los segmentos significativos (citas). Los segmentos se identifican con palabras clave o códigos; asimismo, se pueden escribir anotaciones con algunas reflexiones.

Por su lado, el nivel conceptual procura de:

- Comparar las citas a fin de hacer las interpretaciones de las mismas.

- Agrupar los documentos primarios, citas, códigos en familias a fin de ubicarse en un nivel más abstracto en el análisis de los datos.

- Construir redes a fin de vincular y relacionar los diferentes documentos primarios, citas, códigos y familias.

- Realizar anotaciones sobre la interpretación y la construcción de la teoría emergente.

- Redactar el informe que contenga el análisis e interpretación de los resultados obtenidos, junto con la presentación de la construcción de la teoría.

\section{Nvivo}

El software puede ser usado en investigaciones cuantitativas y cualitativas. El proceso requiere de una serie de pasos iterativo en donde se explora, se codifica, se consulta, se reflexiona, se visualiza, se crean memos (González Calatayud, \& Román García, 2016) y el proceso se repite cuantas veces sea necesario hasta que el usuario tenga este satisfecho con los resultados (Valverde Berrocoso, Fernández Sánchez, Sosa Díaz, Garrido Arroyo, Revuelta Domínguez, \& Guerra Antequera, 2017). El software tiene ocho las pestañas y cinco de ellas son consideradas como herramientas ya que las demás se enmarcarían como complementos de estás. De las cinco herramientas dos de ellas son herramientas de inserción, organización y manipulación de datos (crear y datos externos). 
Las tres pestañas restantes (Analizar, Consulta y Explorar) tienen como función el tratamiento de los datos.

En cuanto a las herramientas de analizar, se tiene que estas se encuentran en la pestaña Analizar. La función central de estas herramientas es la codificación y las categorías (Palacios Vicario, Gutiérrez García, \& Sánchez Gómez, 2013). Al respecto, se tienen tres categorías específicas: selección de codificación en, codificar recursos en $y$, codificar en Vivo. la primera categoría se ocupa para codificar en un nuevo nodo; es decir, aquí se crea un nuevo nodo y se realiza la codificación en el mismo. La segunda categoría es la codificación en un nodo que ya se ha creado previamente; es decir, se codifican recursos completos. La tercera categoría consiste en crear un nodo nuevo que toma el nombre del texto seleccionado.

Respecto a las herramientas de consulta, se tiene que ofrecen un enfoque flexible para permite encontrar y analizar palabras o frases (Trigueros Cervantes, Rivera García, Moreno Doña, \& Muñoz Luna, 2016); asimismo como hacer preguntas y encontrar diversos patrones en base a su codificación. Al respecto, el Asistente para Consultas ayudara realizar consultas de búsqueda de texto, consultas frecuencia de palabras, consultas de codificación y consultas de matriz de codificación.

Las herramientas de explorar, se pueden utilizar cuando se hayan ocupado las dos primeras herramientas y, permiten apreciar ciertos resultados del estudio (Valdemoros San Emeterio, Ponce De León Elizondo, \& Sanz Arazuri, 2011). Existen tres categorías: informes, modelos y visualizaciones. La primera categoría contiene información sobre el proyecto. La segunda categoría es una forma de explorar o presentar visualmente los datos del proyecto a fin de revisar las ideas iniciales o teorías sobre el tema e identificar patrones emergentes, teorías y explicaciones.

Las herramientas gráficas, se usan para presentar el proyecto a través de la codificación de recurso, codificación por valor del atributo de un recurso, codificación por valor de atributo de múltiples recursos, codificación para un nodo, entre otros. el software tiene distintos tipos de graficas en 2D y en 3D (gráficos barras, de columna, de pastel, entre otras).

Respecto a las herramientas de análisis de conglomerados, se tiene que se puede utilizar para visualizar los patrones del proyecto por fuentes de agrupación o nodos que comparten palabras similares, valores de atributos similares, o se encuentren codificados de manera similar por los nodos. Aquí los diagramas de análisis Cluster ofrecen una representación gráfica de las fuentes o nodos a fin de apreciar sus similitudes y diferencias.

\section{Conclusiones}

Los cambios tecnológicos son una cuestión palpable en muchos ámbitos de nuestra vida. La forma en que nos comunicamos y aprendemos ha sufrido cambios, por lo que los procesos de investigación no escapan de estos cambios y están sujetos a esta dinámica. Los investigadores deben aprehender a conjugar las prácticas de investigación a la convergencia tecnológica caracterizada por las redes de comunicación a gran escala, acceso a grandes bases de datos y el uso de softwares científicos.

Uno de los momentos más especiales del proceso de investigación es el análisis de datos. Este proceso viene acompañado de una complejidad que se entremezcla con la emoción a fin de vislumbrar algunas respuestas y propuestas sobre el tema en estudio. El análisis de datos es un proceso dinámico, protagónico y transversal en la metodología de la investigación, que conlleva a extraer significados relevantes de los datos obtenidos sobre el problema en estudio. Por lo tanto, en esta fase se da un diálogo continuo entre la teoría y los datos obtenidos para lograr generar conocimientos fundamentados.

Queda en evidencia que el análisis de datos de 
la investigación en ciencias sociales, apoyada en un método cuantitativo, cualitativo o mixto, puede llevarse a cabo con el uso de los softwares científicos. Los softwares tales como el Lisrel, Atlas.ti y Nvivo están pensado como otros instrumentos que pueden emplearse en la investigación y, son las características mismas de cada proyecto, por ejemplo, los objetivos, métodos, contexto, entre otros aspectos, las que van a definir las herramientas (software) que han de utilizarse en el análisis de datos.

Hay que continuar avanzando en el uso de los softwares científicos para el tratamiento de los datos en la investigación en las ciencias sociales a fin de enriquecer el conocimiento con una producción científica caracterizada por un enfoque metodológico que apoye el cuerpo teórico del trabajo $\mathrm{y}$, encamine las futuras investigaciones en esta área de conocimiento. La idea es abrir un proceso de reflexión sobre cómo se está haciendo la investigación en las ciencias sociales y, como se puede plantear la posibilidad de analizar los datos con el uso de un software científico para dar apoyo a los elementos que son propios de una disciplina científica: teoría y método. En el contexto de la investigación, hay que descubrir elementos que permitan dar una discusión crítica sobre el análisis de los datos y los procedimientos seguidos a fin de generar conocimiento, pero apegado, sobre todo, en los softwares científicos a fin de facilitar la comprensión de la realidad estudiada.

\section{Referencias}

Abello Llanos, R. (2008a). Sugerencias sobre el proceso de investigación en ciencias sociales. Barranquilla: Universidad del Norte/DIP.

Abello Llanos, R. (2008b). Sugerencias sobre el proceso de investigación en ciencias sociales. Barranquilla: Universidad del Norte/DIP.

Abello Llanos, R. (Junio, 2009). La investigación en ciencias sociales: sugerencias prácticas sobre el proceso. Investigación \& Desarrollo, 17(1), 208-229.
Arbuckle, J. L. (1995). AMOS user's guide. Chicago: Smallwaters.

Armony, V. (1997). El análisis de datos cualitativos en ciencias sociales: nuevos enfoques y herramientas. Revista de Investigaciones Folclóricas, 12, 9-16.

Babbie, E. (2000). Fundamentos de la Investigación Social, primera edición. México: Thomson Editores.

Balestrini, M. (1998). Estudios documentales, teóricos, análisis de discurso y las historias de vida: una propuesta metodológica para la elaboración de sus proyectos. Caracas: BL Consultores Asociados, Servicio Editorial.

Bentler, P. M. \& Wu, E. J. C. (1995). EQS for Windows: User's guide. Multivariate Software Inc. New York: Encino.

Bonilla Castro, E.; Hurtado Prieto, J. \& Jaramillo Herrera, C. (2009). La investigación. Bogotá: Alfaomega.

Blanco Álvarez, H. (diciembre, 2012). Análisis de datos cualitativos y atlas.ti: una experiencia de formación. Revista Universitaria, 1(1), 103114.

Briones, G. (1998). Métodos y técnicas avanzadas en investigación aplicadas a la educación y a las ciencias sociales, módulo 2 ( $2^{\mathrm{a}}$ ed.). Bogotá: Icfes piie.

Cárdenas Barrios, L. M. \& González Ferro, V. P. (2016, Noviembre). La herramienta informática Atlas.ti en el análisis de fuentes históricas de las prácticas educativas del siglo XIX. Décima Quinta Conferencia Iberoamericana en Sistemas, Cibernética e Informática, Colombia.

Cazau, P. (2006). Introducción a la investigación en ciencias sociales, Tercera Edición. Argentina: Universidad del Museo Social Argentino.

Chacón, E. (2003). Análisis de datos asistido por computador: una herramienta para la 
investigación holística. Medio Internacional, 13, 14-19.

Chacón, E (2016).Software Científico como Soporte para el análisis de datos en la investigación en las ciencias Sociales. , Memorias del I Encuentro Internacional de Matemáticas, pp-35-39.Disponible en: http://docplayer.es/74981471-Encuentrointernacional-en-educacion-matematica.html

Carrasquero, E. \& Chacón, E. (diciembre, 2010). Correspondencia entre los estilos de pensamiento y los estilos gerenciales: una evaluación desde la gerencia educativa. Acción Pedagógica 19 (1), 102-114.

Chacón, E. \& Chacón, C. (diciembre, 2010). Un Modelo para Medir el Sentido de Autoeficacia Docente en Profesores de Inglés como Lengua Extranjera en Secundaria. Revista Evaluar, 10, 1-21.

Crotty, M. (1998). The foundations of social research: Meaning and perspective in the research process. London: Sage.

Cupani, M. (junio, 2012). Análisis de Ecuaciones Estructurales: conceptos, etapas de desarrollo y un ejemplo de aplicación. Revista Tesis, 1, 186199.

Du Toit, S. \& Du Toit, M. (2001). Interactive LISREL: User's Guide. Chicago: Scientific Software International.

Escobedo Portillo, M. T.; Hernández Gómez, J. A.; Estebané Ortega, V. \& Martínez Moreno, G. (Abril, 2016). Modelos de Ecuaciones Estructurales: características, fases, construcción, aplicación y resultados. Ciencia \& Trabajo, 18(55), 16-22).

González Calatayud, V. \& Román García, M. Del M. (Junio, 2016). Investigador novel: estrategias y herramientas en el mundo conectado. Revista Interuniversitaria de Investigación en Tecnología Educativa (RIITE), 0, 95-108.
Hernández Sampieri, R.; Fernández Collado, C. \& Baptista Lucio, P., (2006). Metodología de la investigación (4⿳a ed.). México: Mc Graw- Hill.

Hernández Sánchez, J.; Borjón Robles, E. \& Torres Ibarra, M. (Junio, 2016). Dimensiones de la tecnología en la formación inicial de profesores de matemáticas: un estudio desde el currículum oficial. Eco.Mat, 7(1), 6-19.

Herrera, J. D. (abril, 2010). La investigación en las ciencias sociales: breve historia y retos actuales. Revista de la Universidad de la Salle, 51, 55-70.

Hurtado de Barrera, J. (2000) Metodología de la Investigación Holística Fundación Sypal, Tercera edición, Caracas Venezuela.

Jiménez Becerra, A. (2004). El estado del arte en la investigación en las ciencias sociales. La práctica investigativa en ciencias sociales (29-42). Colombia: Universidad Pedagógica Nacional.

Jöreskog, K. G. \& Sörbom, D. (1996). LISREL 8 User $>$ S reference guide. Chicago: Scientific Software.

Mejía Correa, A. M. (Diciembre, 2009). La investigación en ciencias sociales y humanas bajo el esquema del modelo universidad empresa - Estado: una mirada desde la teoría crítica de la sociedad. Revista Interamericana de Bibliotecología. 32(2), 231-252.

Pabón-Gómez, J. (junio, 2014). Las TICs y la lúdica como herramientas facilitadoras en el aprendizaje de la matemática. Eco.Mat, 5(1), 37-48.

Palacios Vicario, B.; Gutiérrez García, A. \& Sánchez Gómez, M. C. (2013). Nvivo 10: una herramienta de utilidad en el mundo de la comunicación. Ponencia presentada en el $2^{\circ}$ Congreso Nacional sobre Metodología de la Investigación en Comunicación. Salamanca.

Polit, D. \& Hungler, B. (1997). Investigación Científica en Ciencias de la Salud, quinta 
edición. México: Mc Graw Hill Interamericana.

Pérez, S. (1994). Investigación cualitativa. Retos e interrogantes. Tomo II: Técnicas y Análisis de Datos, Primera Edición. España: La Muralla.

Ruiz, M. A.; Pardo, A. \& San Martín, R. (Abril, 2010). Modelos de ecuaciones estructurales. Papeles del Psicólogo, 31(1), 34-45.

Ruiz Palmero, J. (junio, 2011). Herramientas para la investigación en tecnologías de la información y la comunicación. casos de estudio. Profesorado, Revista de Currículum y Formación del Profesorado, 15(1), 139-149.

Rodríguez, R. (2003). Cómo analizar cuantitativamente datos cuantitativos. Argentina: Séller Consulting.

Sabariego Puig, S.; Vilà Baños, R. \& Sandín Esteban, M. P. (Julio, 2014). El análisis cualitativo de datos con ATLAS.ti. Revista d'Innovació i Recerca en Educació, 7(2), 119133.

Sabino, C. (1996). El proceso de investigación. Caracas: Panapo.

San Martín, D. (Junio, 2014). Teoría fundamentada y Atlas.ti: recursos metodológicos para la investigación educativa. Revista Electrónica de Investigación Educativa, 16(1), 104-122.

Servio, A. L. (Marzo, 2016). El análisis de datos cualitativos asistido por programas informáticos. Notas desde experiencias de enseñanza en posgrados en Ciencias Sociales. Revista Latinoamericana de Metodología de la Investigación Social, 10(5), 63-79.

Trigueros Cervantes, C.; Rivera García, E.; Moreno Doña, A. \& Muñoz Luna, R. (Marzo, 2016). Uso del software CAQDAS Nvivo en Ciencias Sociales. Para la investigación con grupos de discusión. Index De Enfermería, 25(3), 171-174).

Varguillas, C. (diciembre, 2006). El uso de atlas.Ti y la creatividad del investigador en el análisis cualitativo de contenido UPEL. Instituto Pedagógico Rural El Mácaro. Laurus, 12, 7387.

Vivas, M.; Chacón, M. \& Chacón, E. (junio, 2010) Competencias socio -emocionales auto percibidas por los futuros docentes. Educere, 014(048), 137-146.

Valdemoros San Emeterio, M. A.; Ponce De León Elizondo, A. \& Sanz Arazuri, E. (Junio, 2011). Fundamentos en el manejo de Nvivo 9 como herramienta al servicio de estudios cualitativos. Contextos Educativos, 14, 11-29.

Valverde Berrocoso, J.; Fernández Sánchez, M. R.; Sosa Díaz, M. J.; Garrido Arroyo, M. Del C.; Revuelta Domínguez, F. I. \& Guerra Antequera, J. (2017). Nuevas ecologías del aprendizaje: análisis de proyectos de aprendizaje trialógico y educación expandida. La Práctica de la investigación cualitativa: ejemplificación de estudios (86-115). Portugal: Ludomedia. 